\title{
Direct capture and electrostatic repulsion in the self-assembly of rare-earth atom superlattices on graphene
}

\author{
Marina Pivetta, Stefano Rusponi, and Harald Brune \\ Institute of Physics, Ecole Polytechnique Fédérale de Lausanne (EPFL), CH-1015 Lausanne, Switzerland
}

(Received 26 July 2017; revised manuscript received 3 January 2018; published 10 September 2018)

\begin{abstract}
We show that the moiré pattern of graphene on $\operatorname{Ir}(111)$ acts as efficient template for the self-assembly of well-ordered superlattices of single rare-earth adatoms. Using Sm and Dy as representative of early and late lanthanides, we observe that the array quality is determined by the deposition temperature and by a large direct capture area, leading to partial dimer formation. These superlattices result from the combination of the inhomogeneous substrate potential for adatom diffusion generated by the moiré and the interatomic electrostatic repulsion originating from electron transfer to graphene. Deposition temperature- and coverage-dependent experiments quantify the energy barriers for adatom diffusion between adjacent graphene lattice sites and between moiré unit cells, as well as the amount of charge transferred to the substrate by each adatom.
\end{abstract}

DOI: 10.1103/PhysRevB.98.115417

\section{INTRODUCTION}

Single atoms at surfaces possess remarkable properties emerging from their low coordination and their interaction with the underlying surface. Single-atom transistors [1] and single-atom magnets [2-5] were reported; single-atom catalysts were proposed [6-9]. These fascinating properties are unique to the respective atom/substrate combination. Regarding magnetism, Ho is a single-atom magnet when adsorbed on $\mathrm{MgO}$ [2], but neither on graphene [10] nor on several metal substrates [11,12]; conversely, Dy shows long spin lifetime when adsorbed on freestandinglike graphene on metals, as for graphene on $\operatorname{Ir}(111)$, while it is paramagnetic when placed directly on $\operatorname{Ir}(111)$ or other metals, on graphene on $\mathrm{Ru}(0001)$, and onto hexagonal boron nitride, $h$-BN/Rh(111) $[3,12]$.

For applications, the fabrication of large and well-ordered arrays of single atoms is required for the atom/substrate combinations of interest. A viable route is self-assembly at surfaces [13]. Former examples of self-assembled superlattices of $3 d$ transition metals [14-18] or rare earths [19-22] used long-range adsorbate-adsorbate interactions mediated by surface-state Friedel oscillations on close-packed metal surfaces. However, for the single-atom-magnet systems mentioned above, direct adsorption onto metal substrates is unsuited since long-lived magnetic quantum states require protection against electron and phonon scattering [12,22,23], explaining why thin $\mathrm{MgO}$ or graphene separation layers are needed. Cs atom superlattices were self-assembled on graphene/SiC(0001) [24] and on graphene on $\operatorname{Ir}(111)$ [25], thanks to the combined effect of the graphene moiré pattern and repulsive adatom interactions. Reference [24] suggested that superlattices of single atoms for magnetic storage applications can be created that way. However, Cs is not a stable magnet on graphene.

Dy self-assembles into well-ordered superlattices on graphene on $\operatorname{Ir}(111)$ when deposited at $40 \mathrm{~K}$ [3]. Therefore this system forms a lattice of equidistant atoms where each of them can store information in its bistable magnetic quantum state $J_{z}= \pm 7$, where $z$ labels the out-of-plane direction.
Here we demonstrate superlattice formation also for a second $4 f$ element, namely, Sm, thereby addressing also an early lanthanide. The superlattice formation is triggered by a deposition temperature $T_{\text {dep }}$ that is element dependent. In the available deposition-temperature range $\left(10 \leqslant T_{\text {dep }} \leqslant 78 \mathrm{~K}\right)$, we find $30 \leqslant T_{\text {dep }} \leqslant 70 \mathrm{~K}$ for Dy and $T_{\text {dep }} \geqslant 50 \mathrm{~K}$ for $\mathrm{Sm}$. Detailed investigations of the order parameter and the nearestneighbor distance distribution as a function of deposition temperature, as well as of the relative abundance of monomers, dimers, and larger clusters as a function of coverage, enable one to determine the adatom diffusion barrier, the binding energy difference within the moiré unit cell, the area for direct capture by already adsorbed adatoms, and the charge transfer giving rise to the Coulomb repulsion between the adatoms.

\section{EXPERIMENTAL}

The $\operatorname{Ir}(111)$ single crystal was prepared by $\mathrm{Ar}^{+}$sputtering and annealing cycles. Graphene (gr) was grown by chemical vapor deposition from ethylene (100 L at $1400 \mathrm{~K})$. Dy and Sm were deposited from thoroughly degassed high-purity rods (99.9\%) using an $e$-beam evaporator with a typical flux of $2 \times 10^{-3}$ monolayers (ML) per min, in a background pressure of $1 \times 10^{-10}$ mbar. A coverage $\Theta=1.0 \mathrm{ML}$ is defined as one rare-earth atom per graphene unit cell. The substrate temperature during deposition was chosen as $10 \leqslant T_{\text {dep }} \leqslant 78 \mathrm{~K}$ and the sample was immediately cooled to the scanning tunneling microscopy (STM) operating temperature of 5 K [26] after deposition. The Dy and Sm deposition fluxes were calibrated by counting the number of atoms per unit area in STM images of low coverage and low-deposition-temperature samples. The STM images were acquired in constant current mode with the tunnel voltage $V_{\mathrm{t}}$ applied to the sample.

\section{QUANTIFYING ORDER}

The lattice mismatch between graphene and $\operatorname{Ir}(111)$ leads to the formation of a moiré pattern with a period of 


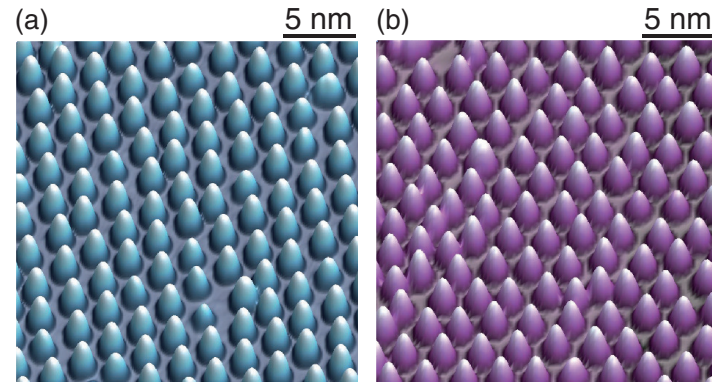

(c)

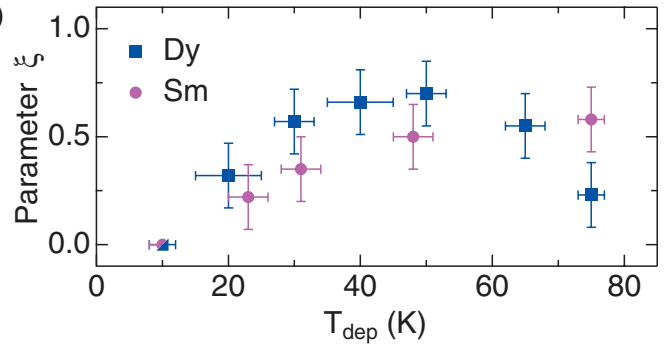

FIG. 1. 3D view of STM images acquired on $0.01 \mathrm{ML}$ of (a) Dy $\left(T_{\text {dep }}=40 \mathrm{~K}, V_{\mathrm{t}}=-200 \mathrm{mV}, I_{\mathrm{t}}=100 \mathrm{pA}\right)$ and (b) Sm on $\mathrm{gr} / \mathrm{Ir}(111)$ $\left(T_{\text {dep }}=48 \mathrm{~K}, V_{\mathrm{t}}=-200 \mathrm{mV}, I_{\mathrm{t}}=50 \mathrm{pA}\right)$. (c) Order parameter $\xi$ as a function of $T_{\mathrm{dep}}$ for both rare-earth elements on $\mathrm{gr} / \operatorname{Ir}(111)$.

approximately ten graphene unit cells [27,28]. Therefore, a coverage of $\Theta=0.01 \mathrm{ML}$ nominally corresponds to one rareearth atom per moiré cell. In Fig. 1 we show the formation of almost perfectly ordered (a) Dy and (b) Sm adatom superlattices obtained for this coverage. The achieved order, however, strongly depends on the deposition temperature. In Fig. 2(a) we show STM images of 0.01 ML of Dy deposited on $\mathrm{gr} / \mathrm{Ir}(111)$ at four temperatures representative of different regimes: random distribution of atoms $\left(T_{\mathrm{dep}}=10 \mathrm{~K}\right)$, partial order $\left(T_{\text {dep }}=20 \mathrm{~K}\right)$, superlattice formation $\left(T_{\text {dep }}=40 \mathrm{~K}\right)$, and partial loss of order $\left(T_{\mathrm{dep}}=75 \mathrm{~K}\right)$. In Fig. 2(b), a similar series is shown for $0.01 \mathrm{ML}$ of Sm: random distribution of atoms $\left(T_{\mathrm{dep}}=10 \mathrm{~K}\right)$, atoms more homogeneously distributed but without order $\left(T_{\mathrm{dep}}=23 \mathrm{~K}\right)$, superlattice formation $\left(T_{\mathrm{dep}}=\right.$ $48 \mathrm{~K}$ ), and appearance of some larger objects and holes $\left(T_{\text {dep }}=75 \mathrm{~K}\right)$.

To quantify the degree of spatial order as a function of deposition temperature, we use a procedure proposed for superlattices of three-dimensional (3D) nanocrystals [29] and formerly applied by us to Sm clusters [30]. The procedure is based on the analysis of the radial decay of the twodimensional (2D) autocorrelation function (2D-ACF) derived from microscopy images. To describe the decay of the envelop of the one-dimensional (1D) profile we use the function env $(r)$ given by the sum of a constant $A_{\infty}$ and two Gaussian functions [29]:

$$
\begin{aligned}
e n v(r)= & A_{\infty}+f_{0} \exp \left(-\frac{1}{2}\left(r / \sigma_{0}\right)^{2}\right) \\
& +f \exp \left(-\frac{1}{2}(r / \sigma)^{2}\right) .
\end{aligned}
$$

The order parameter $\xi$ is defined as

$$
\xi=A_{\infty} /\left(f+A_{\infty}\right)
$$

and ranges from 1 for perfectly ordered superlattices $(f=0)$ to 0 for systems without long-range order. It contains information about the location of the adatoms within the moiré cell, but also about the uniformity of the protrusions, i.e., whether they are all monomers or also dimers and larger clusters. The values of $\xi$ obtained for Dy and $\mathrm{Sm}$ as a function of $T_{\text {dep }}$ are shown in Fig. 1(c). For Dy, good order is obtained for $30 \leqslant T_{\text {dep }} \leqslant 70 \mathrm{~K}$, with best order at $50 \mathrm{~K}$. For $\mathrm{Sm}, \xi$ increases monotonously in the investigated temperature range, reaching values higher than 0.5 at $50 \mathrm{~K}$. We consider that $\xi>0.5$ is indicative of very good order. This is inferred from an extensive analysis carried out on many systems reported in the literature [30]. Clearly, the transition is smooth and the degree of order increases gradually when going towards larger $\xi$ values. The error bars reported on $\xi$ take into account the values obtained for different STM images acquired on the same sample. The error bars on $T_{\text {dep }}$ take into account the variation of the sample temperature during the deposition. In fact, in our setup it is not possible to fully stabilize the temperature during the preparation; therefore $T_{\text {dep }}$ can vary slightly during the deposition, with a maximum variation of $\pm 2 \mathrm{~K}$ over a deposition time of $300 \mathrm{~s}$.

\section{ADSORPTION SITE IN THE MOIRÉ CELL}

Moiré cells comprise atop, hollow hcp and fcc, and transition stacking regions with respect to the $\operatorname{Ir}(111)$ substrate $[27,28]$. In atop regions, the center of a graphene carbon ring is on top of a surface Ir atom; in hcp and fcc regions the carbon ring is centered on top of an hcp or fcc site, respectively. The relative appearance of these different regions in the STM images depends on the tunneling parameters and on the tip [28]. In our measurements the atop stacking areas of the moiré are always imaged as circular depressions, although they protrude geometrically [3]. Surrounding hcp and fcc regions show up as brighter regions having similar contrast.

Figure 3(a) shows an STM image acquired on 0.01 ML Dy on $\mathrm{gr} / \mathrm{Ir}(111)$ for Dy deposition at $T_{\text {dep }}=70 \mathrm{~K}$. Part of the moiré cells are empty owing to strongly increased dimer formation at this temperature, and the moiré contrast is visible. We deduce that the Dy atoms (the round, brightest protrusions in the image) and most of the dimers (slightly elongated, dimmer objects) preferentially adsorb on the atop stacking region of the moiré. For Sm, we show in Fig. 3(b) a sample prepared at $T_{\text {dep }}=78 \mathrm{~K}$. Most of the cells are empty thanks to the low coverage of $2.5 \times 10^{-3} \mathrm{ML}$. Similar to Dy, the Sm adatoms occupy the atop regions of the moiré. Note that transition metals preferentially adsorb on hcp regions [27], indicating a different adsorption mechanism than for rare earths. Calculations show that rare earths on freestanding graphene present similarities with alkali metals, with a bond that is mainly ionic and the charge transferred from the atom to the graphene delocalized over several graphene cells [31]. For Cs on $\operatorname{gr} / \operatorname{Ir}(111)$, density functional theory (DFT) calculations found that adsorption is favored in the vicinity of the atop region of the moiré cell [25].

\section{MECHANISM FOR SUPERLATTICE FORMATION}

\section{A. Statistical analysis}

Analysis of the apparent height of the protrusions in the STM images acquired on Dy/gr/Ir(111) reveals the 


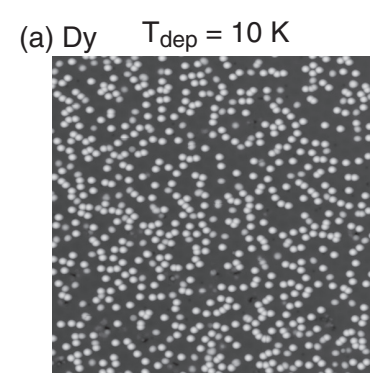

(b) $\mathrm{Sm} \quad \mathrm{T}_{\text {dep }}=10 \mathrm{~K}$

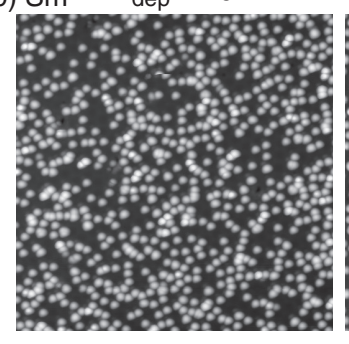

$\mathrm{T}_{\text {dep }}=30 \mathrm{~K}$

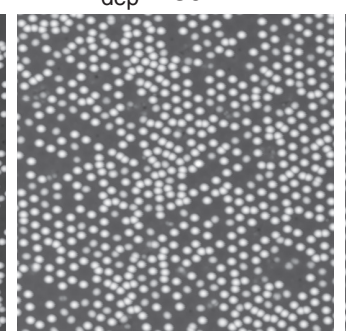

$\mathrm{T}_{\text {dep }}=23 \mathrm{~K}$

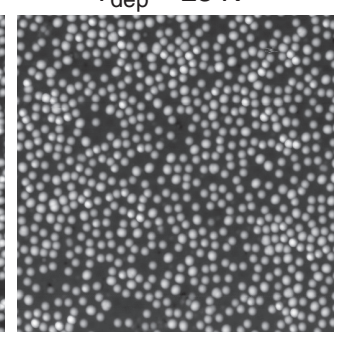

$\mathrm{T}_{\text {dep }}=40 \mathrm{~K}$

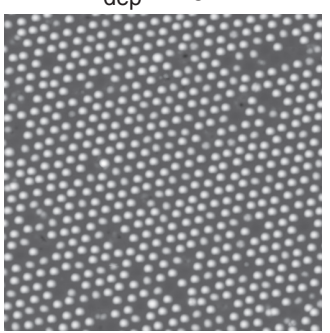

$\mathrm{T}_{\text {dep }}=48 \mathrm{~K}$

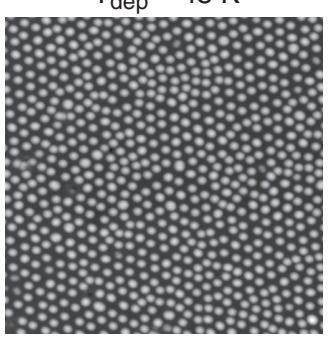

$\mathrm{T}_{\text {dep }}=75 \mathrm{~K}$

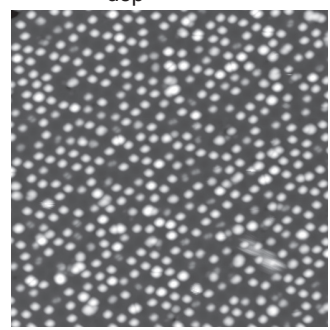

$\mathrm{T}_{\text {dep }}=75 \mathrm{~K}$

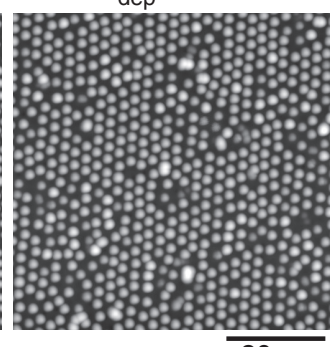

$20 \mathrm{~nm}$

FIG. 2. STM images acquired on (a) $(1.0 \pm 0.1) \times 10^{-2} \mathrm{ML}$ of Dy and (b) $(1.0 \pm 0.1) \times 10^{-2} \mathrm{ML}$ of $\mathrm{Sm}$ on $\mathrm{gr} / \mathrm{Ir}(111)$ for the indicated $T_{\text {dep }}$. Typical tunneling parameters are $V_{\mathrm{t}}=-500 \mathrm{mV}, I_{\mathrm{t}}=20 \mathrm{pA}$.

presence of mainly two species identified as Dy monomers and dimers. Dimers are characterized by lower apparent height and slightly elongated shape, and can be split into two monomers by applying a voltage ramp [3]. The proportion of monomers, dimers, and larger clusters as a function of $T_{\text {dep }}$ is shown in Fig. 4(a). Interestingly, for deposition temperatures between 10 and $50 \mathrm{~K}$, the relative amount of monomers and dimers is constant within the error bar. It equals $(83 \pm 4) \%$ and $(17 \pm 3) \%$, respectively. For higher $T_{\text {dep }}$ the proportion of monomers decreases, mainly owing to formation of additional dimers. This behavior can be rationalized by considering that up to $50 \mathrm{~K}$ Dy dimers are created exclusively by impingement within a capture area around an already adsorbed atom. The high proportion of dimers at low coverage suggests that the capture area is quite large. Only for higher temperatures, dimers form by lateral attachment of adatoms diffusing toward each other from far distances. This is indicative of a repulsion
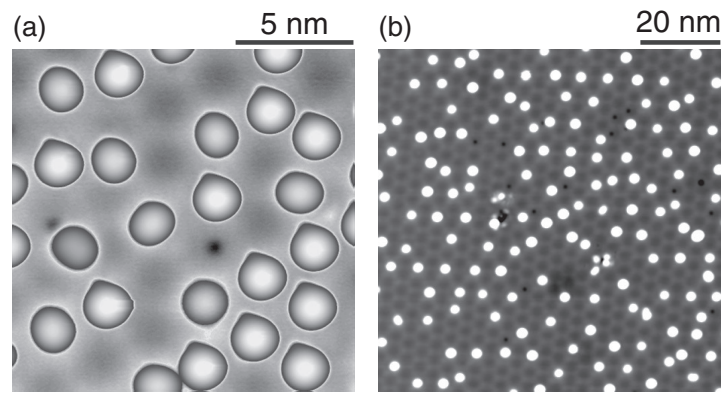

FIG. 3. (a) STM image of $1 \times 10^{-2}$ ML Dy on $\operatorname{gr} / \operatorname{Ir}(111)\left(T_{\text {dep }}=\right.$ $70 \mathrm{~K}, V_{\mathrm{t}}=-400 \mathrm{mV}, I_{\mathrm{t}}=20 \mathrm{pA}$ ). (b) STM image of $2.5 \times 10^{-3}$ ML Sm on $\operatorname{gr} / \operatorname{Ir}(111)\left(T_{\text {dep }}=78 \mathrm{~K}, V_{\mathrm{t}}=-300 \mathrm{mV}, I_{\mathrm{t}}=50 \mathrm{pA}\right)$. Gray scale is adapted in order to enhance the moiré corrugation. Some adsorbates and defects in the graphene layer are visible as black spots. between the atoms that can only be overcome for $T>50 \mathrm{~K}$. This repulsion is very likely electrostatic, since rare-earth atoms, similar to alkali atoms [24,31], are expected to transfer electrons to graphene $[25,31,32]$, resulting in positively charged adatoms.

To determine the capture area for dimer formation by direct impingement, we have performed coverage-dependent measurements for Dy, with $\Theta$ between $2 \times 10^{-3}$ and $2 \times$ $10^{-2} \mathrm{ML}$, once for statistical growth conditions $\left(T_{\mathrm{dep}}=10 \mathrm{~K}\right)$ and once for superlattices $\left(T_{\mathrm{dep}}=40-50 \mathrm{~K}\right)$. The relative abundance of monomers, dimers, and bigger clusters is reported in Fig. 4(b). We use a binomial distribution to describe the statistical formation of dimers and clusters as a function of coverage in the absence of adatom diffusion. We associate to each adatom a direct impingement area constituted of $n$ graphene unit cells such that landing of an additional atom in that area leads to the formation of a dimer or of a larger cluster. The probability $P$ of finding $k$ atoms $(k=0,1,2, \ldots)$ in the area occupied by $n$ cells as a function of the adatom coverage $\Theta$ is given by [33]

$$
P(k, n, \Theta)=\left(\begin{array}{l}
n \\
k
\end{array}\right) \Theta^{k}(1-\Theta)^{n-k} .
$$

In Table I we report the monomer, dimer, and bigger cluster populations calculated for different impingement areas

TABLE I. Monomer, dimer, and bigger cluster populations (percentage) calculated for direct impingement areas of $n$ equal to 7, 19, 31,37 , and 43 graphene sites for $\Theta=0.01 \mathrm{ML}$.

\begin{tabular}{lcrrrr}
\hline \hline$n$ & 7 & 19 & 31 & 37 & \multicolumn{1}{c}{43} \\
\hline Monomers & 97.0 & 91.0 & 85.4 & 83.2 & 80.6 \\
Dimers & 3.0 & 8.0 & 13.1 & 15.2 & 17.1 \\
Clusters & 0 & 1.0 & 1.5 & 1.6 & 2.3 \\
\hline \hline
\end{tabular}



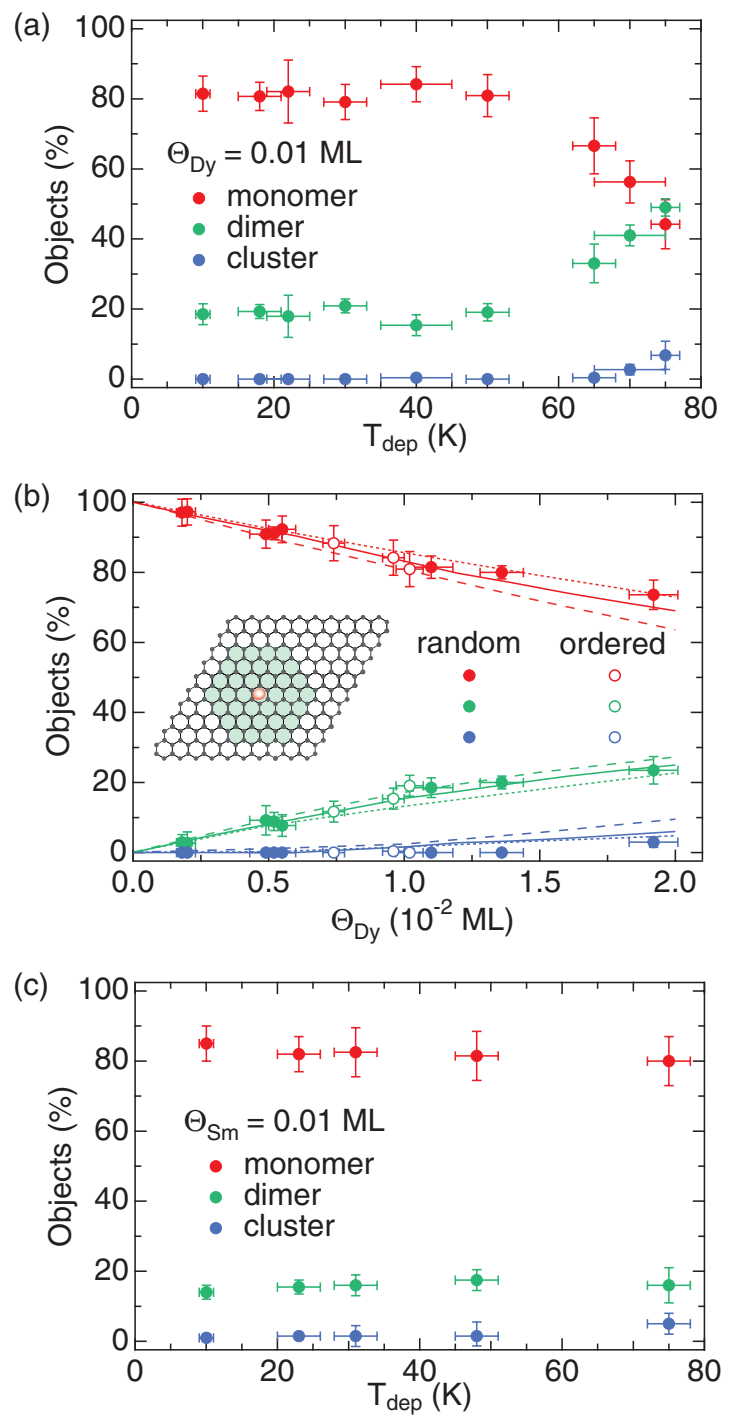

FIG. 4. (a) Relative abundance of monomers, dimers, and larger clusters for $(1.0 \pm 0.1) \times 10^{-2}$ ML Dy on $\operatorname{gr} / \operatorname{Ir}(111)$ as a function of $T_{\text {dep. }}$ (b) Same abundances as a function of Dy coverage for statistical growth $\left(T_{\text {dep }}=10 \mathrm{~K}\right.$, full symbols $)$ and ordered superlattices $\left(T_{\text {dep }}=\right.$ 40 or $50 \mathrm{~K}$, open symbols). Lines: binomial distributions calculated for differently sized direct impingement areas for dimer or cluster formation ( 37 graphene sites full, 31 dotted, and 43 dashed line). Inset: Direct impingement area of $37 \mathrm{gr}$ sites (green) on $(10 \times 10)$ moiré cell. (c) As in (a), but for Sm.

expressed in graphene sites for $\Theta=0.01 \mathrm{ML}$. The obtained values demonstrate that a small impingement area does not account for the populations observed experimentally for Dy on $\mathrm{gr} / \mathrm{Ir}(111)$ shown in Fig. 4(b). In order to describe them, a much larger direct impingement area has to be considered. The observed abundances can very well be reproduced by a binomial distribution with an impingement area for dimer and cluster formation of $n=37 \pm 6$ graphene sites [see inset in Fig. 4(b)] corresponding to $r_{\mathrm{c}} \approx 3 a_{\mathrm{gr}}$, where $a_{\mathrm{gr}}$ is the graphene lattice parameter. Values for $n=31,37$, and 43 are reported in Fig. 4(b), showing also very good agreement with the observed coverage dependence. This large capture area is detrimental to the formation of single-atom superlattices as it
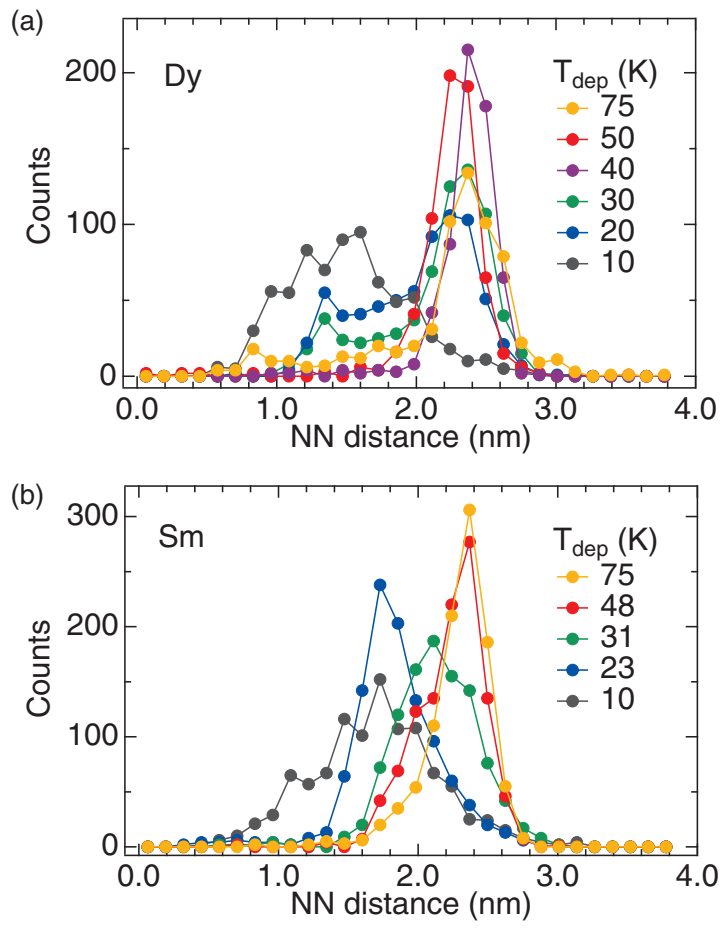

FIG. 5. Histogram of nearest neighbor (NN) distances for $0.01 \mathrm{ML}$ of (a) Dy and (b) Sm monomers and dimers as a function of $T_{\text {dep }}$. Each dot represents the bin center, lines are drawn as guide to the eye.

unavoidably leads to a certain amount of dimers. Notice that a similar impingement area for dimer formation was reported for $\mathrm{Er}$ on $\mathrm{Cu}(111)$ [22].

Figure 4(c) presents the relative abundance of monomers, dimers, and larger clusters for 0.01 ML Sm on $\mathrm{gr} / \mathrm{Ir}(111)$ as a function of $T_{\text {dep }}$. For deposition temperatures between 10 and $75 \mathrm{~K}$, the relative amount of monomers and dimers is fairly constant within the error bar, equal to $82 \pm 4 \%$ and $18 \pm 3 \%$, respectively, but for the deposition at $75 \mathrm{~K}$ we detect the formation of larger clusters. Comparing this result with the one obtained for Dy, we can deduce that (i) the direct impingement area for dimer formation is similar for the two rare earths, and (ii) the repulsive barrier for dimer formation by diffusion is higher for Sm than for Dy.

\section{B. One-dimensional model}

The parameters at the origin of superlattice formation are the adatom diffusion barriers between adjacent graphene lattice sites $\left(E_{\mathrm{d}}\right)$, the adsorption energy difference between different stacking areas of the moire $\left(E_{\mathrm{m}}\right)$, as well as the mutual repulsion between the adatoms $\left(E_{q}\right)$. In order to quantify all three parameters, we have analyzed nearest-neighbor (NN) distance distributions as a function of $T_{\mathrm{dep}}$. For Dy, the results for six deposition temperatures, including the data shown in Fig. 2, are presented in Fig. 5(a). For $T_{\text {dep }}=10 \mathrm{~K}$, the shortest observed NN distance $\left(d_{\min }\right)$ is $0.8 \mathrm{~nm}$. This is in line with our previous observation, that an atom landing at $r \leqslant r_{\mathrm{c}}(\approx 0.75 \mathrm{~nm})$ from an adatom forms a dimer with it. The NN distributions found for $T_{\text {dep }}=20$ and $30 \mathrm{~K}$ show a larger value for $d_{\text {min }} \approx 1.2 \mathrm{~nm}$ and a peak at $2.4 \mathrm{~nm}$. From 
(a)
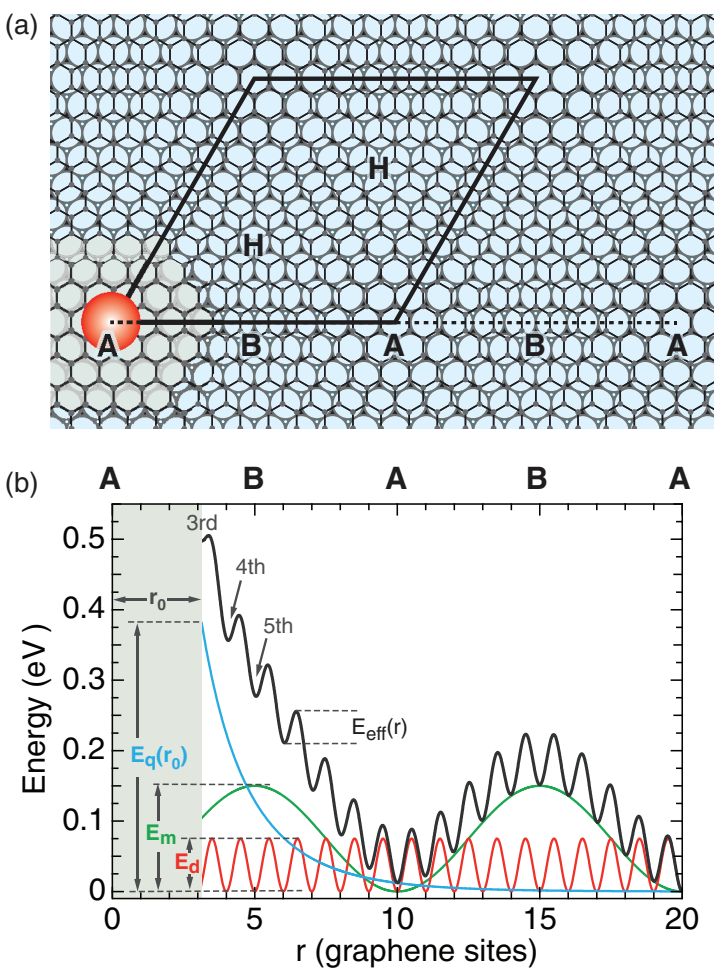

FIG. 6. (a) Moiré with $(10 \times 10)$ graphene unit cells on $(9 \times 9)$ $\operatorname{Ir}(111)$ atoms. Stacking areas: (A) atop, (B) bridge, and (H) hollow. Rare-earth atom (red) together with its direct impingement area (gray). (b) 1D binding energy profile (black) for a second rare-earth atom resulting from superposition of atomic (red) and moiré corrugation (green) of graphene, as well as Coulomb repulsion (blue). Values are for Dy.

the first observation we deduce that $d_{\text {min }}$ increases as soon as the Dy atoms can diffuse, indicating a long-range repulsive interaction between the adatoms. From the second observation we infer that already for $T_{\text {dep }}=20 \mathrm{~K}$ part of the Dy atoms can diffuse to the favorable adsorption region in the moiré cell. The peak is at a slightly smaller distance than the moiré periodicity of $2.53 \mathrm{~nm}[27,28]$ due to the finite extension of the potential minimum, combined with the fact that our analysis systematically selects the closest atom. Depositions at $T_{\text {dep }}=$ 40 and $50 \mathrm{~K}$ lead to an $\mathrm{NN}$ distribution peaked at 2.3-2.4 nm, and no other distances are found. Finally, for $T_{\mathrm{dep}}=75 \mathrm{~K}$, where approximately half of the objects present on the surface are dimers, the peak is still present, however, both smaller and larger NN distances are detected. A similar behavior, although with different characteristic temperatures, is observed for $\mathrm{Sm}$ [see Fig. 5(b)]. In particular, the NN distance corresponding to the moiré periodicity appears with $T_{\text {dep }}=31 \mathrm{~K}$ later than for Dy and is maintained at least up to the highest investigated temperature of $75 \mathrm{~K}$.

Based on these observations we can now evaluate $E_{\mathrm{d}}, E_{\mathrm{m}}$, and $E_{q}$ in a simplified 1D potential energy model. Figure 6(a) shows a schematic representation of the graphene moiré on $\operatorname{Ir}(111)$. A moiré unit cell is indicated, with the different stacking regions defined with the center of the graphene $\mathrm{C}_{6}$ ring being located close to on-top substrate atoms (A), close to substrate bridge sites (B), and close to substrate hollow sites
TABLE II. Diffusion barrier $E_{\mathrm{d}}$, moiré barrier $E_{\mathrm{m}}$, repulsive term $E_{q}$ at the distance $r_{0}=3 a_{\mathrm{gr}}$, and charge transfer $q$ deduced from $E_{\mathrm{q}}\left(r_{0}\right)$. Barriers are given in $\mathrm{meV}$, charge in electron. Values derived from NN distance distributions of Fig. 5, using $v_{0}=10^{13} \mathrm{~Hz}$ and $v=1 \mathrm{~Hz}$. Error bars on the energies correspond to ranges of values, providing a satisfactory description of the experimental data.

\begin{tabular}{lrccc}
\hline \hline & \multicolumn{1}{c}{$E_{\mathrm{d}}$} & $E_{\mathrm{m}}$ & $E_{q}\left(r_{0}\right)$ & $q$ \\
\hline Dy & $75 \pm 5$ & $150 \pm 30$ & $375 \pm 30$ & $0.74 \pm 0.03$ \\
Sm & $100 \pm 5$ & $160 \pm 30$ & $480 \pm 35$ & $0.83 \pm 0.03$ \\
\hline \hline
\end{tabular}

(H). The most favorable adsorption site within the moiré cell for both species is the atop region (see Fig. 3). In addition, we have shown that for Dy the atomic adsorption site is the sixfold-coordinated hollow site [3]. We simplify the potential landscape induced by the moiré by attributing a global minimum in the atop stacking area and the same height to hollow and bridge stacked regions. The repulsion is described by a screened Coulomb potential [34,35] since a pure Coulomb potential turns out to be too long-ranged, and dipolar interactions are too weak at the relevant distances, as estimated from dipole moments calculated for rare-earth atoms [31,32] and alkali metals $[31,34]$ on freestanding graphene. The screening effect is due to the redistribution of the electrons in graphene around the charged adatoms, yielding a lower effective charge for the repulsion and a faster decay. Such a screened potential is usually expressed as

$$
E_{q}(r)=\frac{1}{4 \pi \epsilon_{0}} \frac{q^{2}}{r} \exp \left(-\frac{r}{r_{0}}\right),
$$

where $q$ is the charge transferred and $r_{0}$ is the screening length. To model the potential experienced by the adatoms, these two quantities are considered as free parameters. Our data are best described using $r_{0}=0.75 \mathrm{~nm}$, i.e., the screening length is of the order of three graphene unit cells. From the amplitude of the repulsive term we deduce the charge transfer $q$ (electrons) reported in Table II for Dy and Sm. The resulting 1D potential felt by an adatom as a function of its distance from an adatom adsorbed in the center of an atop region is shown as a full black line in Fig. 6(b).

It remains to define a time scale in which the lattice forms and assume an attempt frequency for diffusion, for which we take the expected value of $v_{0}=10^{13} \mathrm{~Hz}$ [13]. We see no difference between lattices held for hours at the deposition temperature and those that were cooled down immediately after deposition, reaching $10 \mathrm{~K}$ after $5 \mathrm{~min}$ and the equilibrium temperature of $5 \mathrm{~K}$ after $1 \mathrm{~h}$. Therefore the superlattice formation takes place during deposition and there is no postevolution. At the employed flux and coverage, deposition takes typically $300 \mathrm{~s}$ and all diffusion steps have to be fast compared to this time. As can be seen in Fig. 6, the diffusion barrier of each individual jump $E_{\text {eff }}$ depends on the initial site and the jump direction. Thus, we adjust $E_{\mathrm{d}}, E_{\mathrm{m}}$, and $E_{q}$ to give the observed temperature-dependent $\mathrm{NN}$ distributions shown in Fig. 5(a) with the constraint that $v=v_{0} \exp \left(-E_{\text {eff }} / \mathrm{k}_{\mathrm{B}} T\right) \geqslant$ $1 \mathrm{~Hz}$ for any activated process. For comparison, requiring a frequency of $100 \mathrm{~Hz}$ lowers the barriers by $15 \%$. Note also that in our simplified 1D model we compare the height 

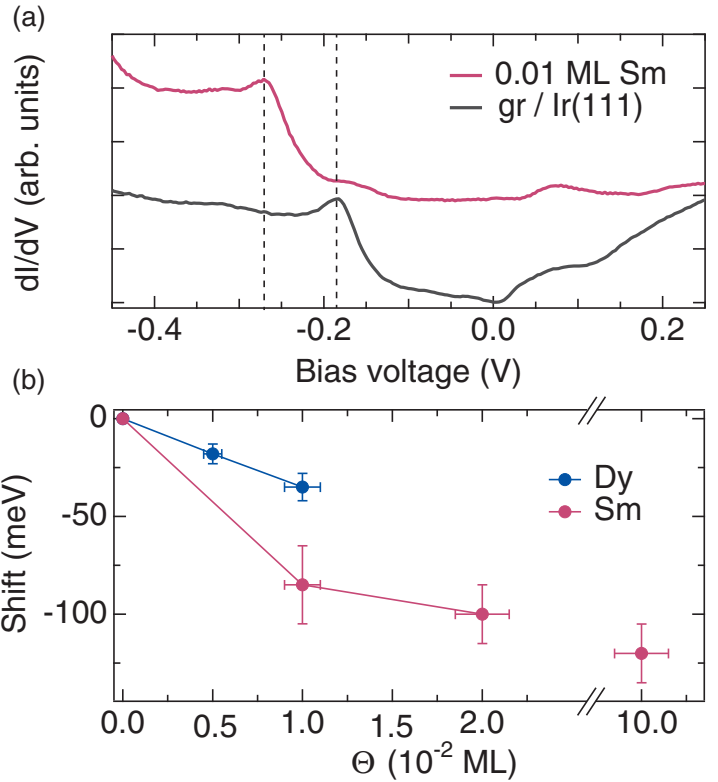

FIG. 7. (a) $d I / d V$ spectra acquired on bare gr/Ir(111) (gray) and on $\mathrm{gr} / \mathrm{Ir}(111)$ in the presence of $0.01 \mathrm{ML}$ of Sm (magenta). (b) Energy shift of the $\operatorname{Ir}(111)$ surface state as a function of Sm and Dy coverage.

of the effective barriers, while in the real 2D case also the multiplicity of each process should be taken into account. As a result, the $1 \mathrm{D}$ model tends to overestimate the energy barriers. However, the hierarchy between the different energies and the processes leading to the superlattice formation are not affected by the dimensionality of the model $[36,37]$.

$E_{\mathrm{d}}, E_{\mathrm{m}}$, and $E_{q}$ are interdependent and contribute to define the activation temperature of the different diffusion processes. Diffusion inside a moiré cell towards the most favorable adsorption site takes place at $20 \mathrm{~K}$ for Dy and at $30 \mathrm{~K}$ for $\mathrm{Sm}$. Diffusion between moiré cells sets in at $40 \mathrm{~K}$ for Dy and at $50 \mathrm{~K}$ for $\mathrm{Sm}$. These temperatures mainly define $E_{\mathrm{d}}$ and $E_{\mathrm{m}}$. The amplitude and the extension of the screened Coulomb potential can be deduced from the fact that Dy and Sm atoms are able to move from the 4th to the 5th graphene site around a previously adsorbed atom at $20 \mathrm{~K}$ but not at $10 \mathrm{~K}$. This process is responsible for the larger values of $d_{\min }$ observed for $T_{\text {dep }} \approx 20 \mathrm{~K}$, [see Figs. 5(a) and 5(b)]. In addition, the potential must reproduce the observation that adatoms can laterally penetrate the direct impingement area and form dimers for Dy at $60 \mathrm{~K}$ and for Sm above $75 \mathrm{~K}$ (Fig. 4). The rate-limiting step is from the 4th to the 3rd graphene site around another atom. All together, these observations determine the values for the energies $E_{\mathrm{d}}, E_{\mathrm{m}}$, and $E_{q}$ given in Table II for each of the two elements, as well as the screening radius $r_{0}=3 a_{\mathrm{gr}}$. Note that $E_{q}$ and $r_{0}$ allow one to estimate the amount of charge $q$ transferred from the adatoms to graphene, also reported in Table II.

DFT results are available for atoms on freestanding graphene, which we consider a reasonable assumption for $\mathrm{gr} / \operatorname{Ir}(111)$, given the large graphene-Ir(111) mean distance of (338 \pm 4$)$ pm [38], respectively, (339 \pm 3$)$ pm [39]. They yield $E_{\mathrm{d}}=125$ and $326 \mathrm{meV}$ for Dy and Sm [31], respectively, which is clearly higher than our values but exhibits the same trend for the two species. Concerning the charge transfer, the DFT calculations [31] yield a transfer of 0.77 electrons for Dy, in very good agreement with what we estimate, and 0.65 electrons for $\mathrm{Sm}$, smaller than our value.

The existence of charge transfer is also supported by our $d I / d V$ measurements, showing a shift of the Ir surface state $[40,41]$ towards higher binding energies in the presence of Dy or Sm on $\mathrm{gr} / \mathrm{Ir}(111)$. The $d I / d V$ spectrum acquired on pristine $\mathrm{gr} / \mathrm{Ir}(111)$ is shown in Fig. 7(a) and presents a peak at $-180 \mathrm{mV}$ corresponding to the $\operatorname{Ir}(111)$ surface state modified by the graphene layer $[40,41]$. In the presence of Sm or Dy we observe a shift of this peak towards higher binding energies, as shown for the case of $0.01 \mathrm{ML}$ Sm. Figure 7(b) presents the energy shift as a function of Sm and Dy coverage. From these data we deduce that both Sm and Dy transfer electrons to graphene, and that the transfer is larger for Sm than for Dy, as for equal coverage the energy shift is larger for $\mathrm{Sm}$. This is in qualitative agreement with the values deduced from the interaction strength of the screened Coulomb potential. Quantitative values cannot be inferred from the surface-state shift shown in Fig. 7(b) since the mechanism of that shift is not well understood [41].

\section{CONCLUSIONS}

By using Sm and Dy as prototypes, we have elucidated the mechanisms leading to the formation of rare-earth atom superlattices on graphene on $\operatorname{Ir}(111)$. Deposition temperatureand coverage-dependent experiments allow us to identify the ordering mechanism as being a combination of inhomogeneous substrate potential and Coulomb repulsion. Dy is a single-atom magnet, i.e., information can be stored magnetically in each individual atom. If large coherence times are found for rare-earth atoms on graphene, they may even serve as magnetic quantum bits.
[1] M. Fuechsle, J. A. Miwa, S. Mahapatra, H. Ryu, S. Lee, O. Warschkow, L. C. L. Hollenberg, G. Klimeck, and M. Y. Simmons, A single-atom transistor, Nat. Nanotechnol. 7, 242 (2012).

[2] F. Donati, S. Rusponi, S. Stepanow, C. Wäckerlin, A. Singha, L. Persichetti, R. Baltic, K. Diller, F. Patthey, E. Fernandes, J. Dreiser, Ž. Šljivančanin, K. Kummer, C. Nistor, P. Gambardella, and $\mathrm{H}$. Brune, Magnetic remanence in single atoms, Science 352, 318 (2016).
[3] R. Baltic, M. Pivetta, F. Donati, C. Wäckerlin, A. Singha, J. Dreiser, S. Rusponi, and H. Brune, Superlattice of single atom magnets on graphene, Nano Lett. 16, 7610 (2016).

[4] F. D. Natterer, K. Yang, W. Paul, P. Willke, T. Choi, T. Greber, A. J. Heinrich, and C. P. Lutz, Reading and writing single-atom magnets, Nature (London) 543, 226 (2017).

[5] F. D. Natterer, F. Donati, F. Patthey, and H. Brune, Thermal and Magnetic-Field Stability of Holmium Single-Atom Magnets, Phys. Rev. Lett. 121, 027201 (2018). 
[6] A. S. Wörz, K. Judai, S. Abbet, J. M. Antonietti, U. Heiz, A. Del Vitto, L. Giordano, and G. Pacchioni, Chemistry on single atoms: Key factors for the acetylene trimerization on MgO-supported Rh, Pd, and Ag atoms, Chem. Phys. Lett. 399, 266 (2004).

[7] X.-F. Yang, A. Wang, B. Qiao, J. Li, J. Liu, and T. Zhang, Single-atom catalysts: A new frontier in heterogeneous catalysis, Acc. Chem. Res. 46, 1740 (2013).

[8] G. S. Parkinson, Z. Novotny, G. Argentero, M. Schmid, J. Pavelec, R. Kosak, P. Blaha, and U. Diebold, Carbon monoxideinduced adatom sintering in a $\mathrm{Pd}-\mathrm{Fe}_{3} \mathrm{O}_{4}$ model catalyst, Nat. Mater. 12, 724 (2013).

[9] R. Bliem, J. van der Hoeven, A. Zavodny, O. Gamba, J. Pavelec, P. E. de Jongh, M. Schmid, U. Diebold, and G. S. Parkinson, An atomic-scale view of $\mathrm{CO}$ and $\mathrm{H}_{2}$ oxidation on a $\mathrm{Pt} / \mathrm{Fe}_{3} \mathrm{O}_{4}$ model catalyst, Angew. Chem., Int. Ed. Engl. 54, 13999 (2015).

[10] R. Baltic, F. Donati, A. Singha, C. Wäckerlin, J. Dreiser, B. Delley, M. Pivetta, S. Rusponi, and H. Brune, Magnetic properties of single rare-earth atoms on graphene/Ir(111), Phys. Rev. B 98, 024412 (2018).

[11] F. Donati, A. Singha, S. Stepanow, C. Wäckerlin, J. Dreiser, P. Gambardella, S. Rusponi, and H. Brune, Magnetism of Ho and Er Atoms on Close-Packed Metal Surfaces, Phys. Rev. Lett. 113, 237201 (2014).

[12] A. Singha, R. Baltic, F. Donati, C. Wäckerlin, J. Dreiser, L. Persichetti, S. Stepanow, P. Gambardella, S. Rusponi, and H Brune, $4 f$ occupancy and magnetism of rare-earth atoms adsorbed on metal substrates, Phys. Rev. B 96, 224418 (2017).

[13] H. Brune, Microscopic view of epitaxial metal growth: Nucleation and aggregation, Surf. Sci. Rep. 31, 125 (1998).

[14] J. Repp, F. Moresco, G. Meyer, K. H. Rieder, P. Hyldgaard, and M. Persson, Substrate Mediated Long-Range Oscillatory Interaction Between Adatoms: $\mathrm{Cu} / \mathrm{Cu}(111)$, Phys. Rev. Lett. 85, 2981 (2000).

[15] N. Knorr, H. Brune, M. Epple, A. Hirstein, M. A. Schneider, and K. Kern, Long-range adsorbate interactions mediated by a two-dimensional electron gas, Phys. Rev. B 65, 115420 (2002).

[16] N. N. Negulyaev, V. S. Stepanyuk, L. Niebergall, P. Bruno, W. Auwärter, Y. Pennec, G. Jahnz, and J. V. Barth, Effect of strain relaxations on heteroepitaxial metal-on-metal island nucleation and superlattice formation: $\mathrm{Fe}$ on $\mathrm{Cu}(111)$, Phys. Rev. B 79, 195411 (2009).

[17] X. P. Zhang, B. F. Miao, L. Sun, C. L. Gao, A. Hu, H. F. Ding, and J. Kirschner, Atomic superlattice formation mechanism revealed by scanning tunneling microscopy and kinetic Monte Carlo simulations, Phys. Rev. B 81, 125438 (2010).

[18] M. Pivetta, G. E. Pacchioni, U. Schlickum, J. V. Barth, and H. Brune, Formation of Fe Cluster Superlattice in a Metal-Organic Quantum-Box Network, Phys. Rev. Lett. 110, 086102 (2013).

[19] F. Silly, M. Pivetta, M. Ternes, F. Patthey, J. P. Pelz, and W.-D. Schneider, Creation of an Atomic Superlattice by Immersing Metallic Adatoms in a Two-Dimensional Electron Sea, Phys. Rev. Lett. 92, 016101 (2004).

[20] N. N. Negulyaev, V. S. Stepanyuk, L. Niebergall, P. Bruno, M. Pivetta, M. Ternes, F. Patthey, and W.-D. Schneider, Melting of Two-Dimensional Adatom Superlattices Stabilized by LongRange Electronic Interactions, Phys. Rev. Lett. 102, 246102 (2009).

[21] R. X. Cao, X. P. Zhang, B. F. Miao, Z. F. Zhong, L. Sun, B. You, A. Hu, and H. F. Ding, Self-organized Gd atomic superlattice on $\operatorname{Ag}(111)$ : Scanning tunneling microscopy and kinetic Monte Carlo simulations, Surf. Sci. 610, 65 (2013).

[22] A. Singha, F. Donati, C. Wäckerlin, R. Baltic, J. Dreiser, M. Pivetta, S. Rusponi, and H Brune, Magnetic hysteresis in Er trimers on $\mathrm{Cu}(111)$, Nano Lett. 16, 3475 (2016).

[23] A. A. Khajetoorians, S. Lounis, B. Chilian, A. T. Costa, L. Zhou, D. L. Mills, J. Wiebe, and R. Wiesendanger, Itinerant Nature of Atom-Magnetization Excitation by Tunneling Electrons, Phys. Rev. Lett. 106, 037205 (2011).

[24] C.-L. Song, B. Sun, Y.-L. Wang, Y.-P. Jiang, L. Wang, K. He, X. Chen, P. Zhang, X.-C. Ma, and Q.-K. Xue, Charge-TransferInduced Cesium Superlattices on Graphene, Phys. Rev. Lett. 108, 156803 (2012).

[25] M. Petrović, P. Lazić, S. Runte, T. Michely, C. Busse, and M. Kralj, Moiré-regulated self-assembly of cesium adatoms on epitaxial graphene, Phys. Rev. B 96, 085428 (2017).

[26] R. Gaisch, J. K. Gimzewski, B. Reihl, R. R. Schlittler, M. Tschudy, and W. D. Schneider, Low-temperature ultra-high vacuum scanning tunneling microscope, Ultramicroscopy 4244, 1621 (1992).

[27] A. T. N'Diaye, S. Bleikamp, P. J. Feibelman, and T. Michely, Two-Dimensional Ir Cluster Lattice on a Graphene Moiré on Ir(111), Phys. Rev. Lett. 97, 215501 (2006).

[28] A. T. N'Diaye, J. Coraux, T. N. Plasa, C. Busse, and T. Michely, Structure of epitaxial graphene on $\operatorname{Ir}(111)$, New J. Phys. 10, 043033 (2008).

[29] S. Pichler, M. I. Bodnarchuk, M. V. Kovalenko, M. Yarema, G. Springholz, D. V. Talapin, and W. Heiss, Evaluation of ordering in single-component and binary nanocrystal superlattices by analysis of their autocorrelation functions, ACS Nano 5, 1703 (2011).

[30] D. Mousadakos, M. Pivetta, H. Brune, and S. Rusponi, Sm cluster superlattice on graphene/Ir(111), New J. Phys. 19, 123021 (2017).

[31] X. Liu, C. Z. Wang, M. Hupalo, W. C. Lu, M. C. Tringides, Y. X. Yao, and K. M. Ho, Metals on graphene: Correlation between adatom adsorption behavior and growth morphology, Phys. Chem. Chem. Phys. 14, 9157 (2012).

[32] D. F. Förster, T. O. Wehling, S. Schumacher, A. Rosch, and T. Michely, Phase coexistence of clusters and islands: Europium on graphene, New J. Phys. 14, 023022 (2012).

[33] H. Brune, M. Giovannini, K. Bromann, and K. Kern, Selforganized growth of nanostructure arrays on strain-relief patterns, Nature (London) 394, 451 (1998).

[34] X. Liu, C.-Z. Wang, H.-Q. Lin, K. Chang, J. Chen, and K.M. Ho, Charge oscillations and interaction between potassium adatoms on graphene studied by first-principles calculations, Phys. Rev. B 91, 035415 (2015).

[35] P. Löptien, L. Zhou, J. Wiebe, A. A. Khajetoorians, J. L. Mi, B. B. Iversen, Ph. Hofmann, and R. Wiesendanger, Screening and atomic-scale engineering of the potential at a topological insulator surface, Phys. Rev. B 89, 085401 (2014).

[36] M. C. Bartelt and J. W. Evans, Scaling analysis of diffusionmediated island growth in surface adsorption processes, Phys. Rev. B 46, 12675 (1992).

[37] Y. Han, É. Gaudry, T. J. Oliveira, and J. W. Evans, Point island models for nucleation and growth of supported nanoclusters during surface deposition, J. Chem. Phys. 145, 211904 (2016).

[38] C. Busse, P. Lazić, R. Djemour, J. Coraux, T. Gerber, N. Atodiresei, V. Caciuc, R. Brako, A. T. N’Diaye, S. Blügel, 
J. Zegenhagen, and T. Michely, Graphene on $\operatorname{Ir}(111)$ : Physisorption with Chemical Modulation, Phys. Rev. Lett. 107, 036101 (2011).

[39] S. K. Hämäläinen, M. P. Boneschanscher, P. H. Jacobse, I. Swart, K. Pussi, W. Moritz, J. Lahtinen, P. Liljeroth, and J. Sainio, Structure and local variations of the graphene moiré on $\operatorname{Ir}(111)$, Phys. Rev. B 88, 201406 (2013).
[40] A. Varykhalov, D. Marchenko, M. R. Scholz, E. D. L. Rienks, T. K. Kim, G. Bihlmayer, J. Sánchez-Barriga, and O. Rader, Ir(111) Surface State with Giant Rashba Splitting Persists Under Graphene in Air, Phys. Rev. Lett. 108, 066804 (2012).

[41] S. J. Altenburg, J. Kröger, T. O. Wehling, B. Sachs, A. I. Lichtenstein, and R. Berndt, Local Gating of an $\operatorname{Ir}(111)$ Surface Resonance by Graphene Islands, Phys. Rev. Lett. 108, 206805 (2012). 